\title{
|||||||||||||||||||||||||||||||||||||||||||||||||||||||||||||||||||.
}

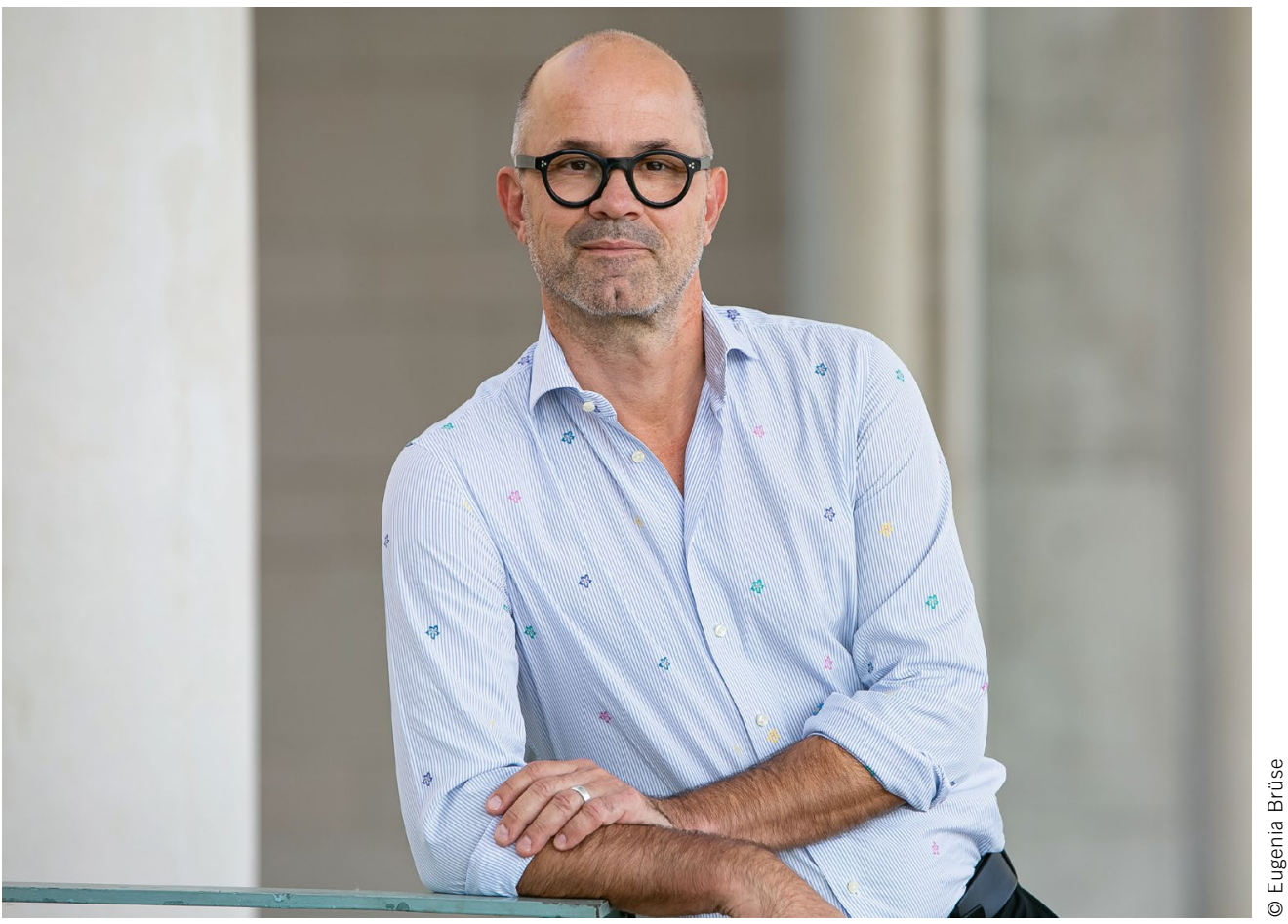

Markus Sigismund

Deputy Head of Department in the Forecasts, Statistics and Special

Surveys division of the German Federal Ministry of Transport and Digital Infrastructure (BMVI)

\section{Mobility - Big Data Isn't always Big}

Every day the people of Germany cover more than three billion kilometers - on foot or by bike, bus, train or car. How can we design our infrastructure to ensure that these journeys are safe, fast and resource-efficient? One essential factor is a detailed understanding of people's mobility behavior.

The differences in the mobility structures and developments linking urban and rural areas, but also within the cities and the countryside present a major challenge. Familiar patterns are starting to disappear. One example of this is that jobs and retail stores are no longer concentrated in the city centers. More and more of the journeys we make have a variety of different destinations. One central obstacle to any research on mobility is the lack of appropriate small-scaled data concerning the interdependencies of transport. We simply don't know how many people are actually traveling from A to B.

In this age of digitalization, the use of Floating Car Data (FCD) and mobile data seems to be an obvious solution, because if these data can be reliably anonymized, they can be used to identify typical mobility structures on a detailed level. This is why more and more frequent calls are being made for the replacement of conventional surveys with big data. However, making a simple paradigm shift of this kind would be premature. In part because of their rapid availability, mobile data provided valuable answers to a wide range of questions during the Covid-19 pandemic. However, they are not yet of sufficiently high quality to allow us to draw representative conclusions about the level and structure of mobility. This is because the data are skewed or, in other words, unrepresentative, as a result of the specific customer and data structure. For example, short journeys are under-recorded in mobile data, because they are not represented accurately within a cell. By contrast, FCD cover only a selection of vehicles. In addition, the data tell us very little about the purposes of the people's journeys, which means that we have almost no information about a factor that plays a crucial role in mobility forecasts.

For this reason, we are currently running a complex research project with the title "VerBindungen" (connections), together with the German Federal Statistical office and other research partners, which aims to bring together the old and the new worlds of data. We are standardizing the FCD and mobile data against traditional data sources and from this synthesis we will be able to obtain reliable data and knowledge of new methods. For this purpose, we can make use of the first small-scale evaluations of commuter data from the German Federal Employment Agency, microdata from the statistical offices on a federal and state level and the surveys carried out by the Federal Ministry of Transport and Digital Infrastructure. Our experiences so far have shown that the hard work is well worthwhile. And the classic data of official statistics and a core of surveys remain indispensable - also for ensuring that big data has big potential. 